\title{
Accuracy of an Electronic Apex Locator in the Retreatment of Teeth Obturated with Plastic or Cross-linked Gutta-percha Carrier-based Materials: An Ex Vivo Study
}

\author{
Manuele Mancini, DDS, Pietro Palopoli, DDS, Lorenzo Iorio, DDS, Gabriele Conte, DDS, \\ and Luigi Cianconi, MD, DDS
}

\section{Ahstract}

Introduction: This ex vivo study evaluated the accuracy of the Root ZX electronic apex locator (EAL) (J Morita Corp, Kyoto, Japan) in determining the working length during retreatment of canals sealed with 2 different carrier-based obturating materials (ProTaper Obturator [Dentsply Maillefer, Ballaigues, Switzerland] and GuttaCore [Dentsply Maillefer]) and also evaluated whether they influenced its accuracy differently. Methods: Fifty extracted single-rooted human teeth with sound apices were selected for the study. We measured canals with the direct visual technique using a \#10 K-file and shaped them with ProTaper Universal instruments (Dentsply Maillefer) up to this predetermined length. After instrumentation, we determined the canal length again using the visual technique (direct length [DL]). This value was considered the "reference point." Specimens were then placed in a tooth holder (Pro-Train; Simit Dental, Mantova, Italy), and the canal length was electronically measured (electronic length 1 [EL1]). Specimens were then obturated with ProTaper Obturators (group 1, $n=25$ ) and GuttaCore (group 2, $n=25$ ). Seven days later, a new electronic length was determined (electronic length 2 [EL2]) during retreatment procedures in the presence of the obturating material. EL1 and EL2 values were compared with DL using the Bland and Altman method. The different influences of the tested materials on the accuracy of the EAL were calculated with repeated measures analysis of variance. Results: Both EL1 and EL2 values provided a statistically significant overestimation of the actual canal length (DL $[P<.05])$, with the EL2 values higher from $D L$. The 2 different materials did not influence the accuracy of the EAL differently $(P=.486)$. Conclusions: The measurements obtained with the EAL tested during orthograde retreatments can lead clinicians to overinstrumentation and consequent overfilling of the endodontic space. (J Endod 2014;40:2061-2065)

\section{Key Words}

Electronic apex locator, GuttaCore, ProTaper Obturator, retreatment, working length

0 ne of the factors that influence the success of root canal treatment is the accurate and complete cleaning of the root canal system without damaging periapical integrity (1). For this goal to be achieved, an accurate working length (WL) should be determined, and cleaning and shaping should be performed to this length (2). Desirable healing is not always provided by the primary endodontic treatment, and when failures occur, retreatment is needed $(3,4)$. Among several alternatives, orthograde nonsurgical retreatment should be the first choice (5-7). Its aim is the complete removal of the root canal filling material to regain access to the apical foramen, thus facilitating both cleaning/shaping of the root canal system and a final proper obturation $(5,6)$. Many different materials have been used for root canal obturation (8), and gutta-percha is the most widely used and accepted (9).

In 1978, Johnson (10) introduced a root canal obturation technique with thermoplasticized gutta-percha on a metallic carrier, which was subsequently substituted by a solid plastic one (11). This obturation device was marketed under the name Thermafil (Dentsply Maillefer, Ballaigues, Switzerland) (10). The system allows for quick and easy placement of thermoplasticized gutta-percha. However, if retreatment is required, it may be more challenging and time-consuming because the carrier and the guttapercha must be removed and the carrier itself is not soluble in common solvents $(12,13)$. To eliminate these drawbacks, the GuttaCore system (Dentsply Maillefer), a carrier-based obturation that uses a cross-linked gutta-percha core instead of plastic carriers, has recently been launched (14). Bergenholtz et al (15) showed the importance of a well-determined WL during retreatment. In fact, in a clinical radiographic follow-up investigation, they showed that the overinstrumentation and overfilling of retreated root canals significantly decreased the frequency of complete biological regeneration of the apical lesion.

Electronic apex locators (EALs) are useful in determining the $\mathrm{WL}$ in primary root canal treatment (16-19) and orthograde retreatment (20). Many studies have evaluated different EALs and factors that may influence their accuracy, such as root canal pre-enlargement, irrigating solutions, solvents, file size, and stage of apex formation (21-23). It has been shown that root canal preparation and root canal filling materials affect root canal impedance (24), which is a sharp change at the apical terminus that is recognized by recent EALs as the apex (25). To our knowledge, few studies have evaluated the accuracy of different EALs during endodontic retreatment of canals obturated with different endodontic materials $(2,20,23,26,27)$. To date, no study has been published evaluating EAL accuracy during retreatment of canals obturated with carrier-based gutta-percha. Therefore, the purpose of this ex vivo evaluation

From the Department of Restorative Dentistry and Endodontics, University of Rome "Tor Vergata", Rome, Italy.

Address requests for reprints to Dr Manuele Mancini, Via Tuscolana 58/64-00182 Rome, Italy. E-mail address: manuele.mancini@tiscali.it 0099-2399/\$ - see front matter

Copyright (C) 2014 American Association of Endodontists. http://dx.doi.org/10.1016/j.joen.2014.07.035 
was 2-fold: to evaluate the accuracy of the EAL Root ZX (J Morita Corp, Kyoto, Japan) during the retreatment of canals obturated with ProTaper Obturator (Dentsply Maillefer) and GuttaCore and to evaluate whether the 2 different obturating materials influenced the accuracy of the tested EAL differently.

\section{Materials and Methods}

Fifty single-rooted straight teeth extracted for periodontal or orthodontic reasons were selected, with the approval of the Ethics in Research Committee of the Centre of Health Science of the University of Rome "Tor Vergata," Rome, Italy. The selected teeth had a single canal and fully formed roots and were devoid of caries, endodontic treatments, and restorations. Specimens were radiographed buccolingually and mesiodistally to identify aberrant canal morphology and to confirm the presence of a single canal.

Teeth were then placed in 5.25\% sodium hypochlorite for 2 hours. Soft tissue and calculus remnants were removed with an ultrasonic scaler (Piezon 5; Castellini, Bologna, Italy). After being cleaned, each root was carefully examined by stereomicroscopy (Universal-300; Moeller-Wedel, Wedel, Germany) at $20 \times$ magnification for the detection of external cracks, open apices, or apices undergoing resorption, which might alter the accuracy of the WL measurements. After microscopic examination, teeth were stored in $0.9 \%$ sodium chloride water solution at room temperature and used within 1 week. Each root was sectioned at a level between 16 and $17 \mathrm{~mm}$ with a crosscut carbide bur (Dentsply Maillefer) in a high-speed handpiece with water spray to produce a flat surface for the precise location of the rubber stop. Conventional endodontic access to the pulp chamber was gained with a round, diamond-coated bur (Dentsply Maillefer). The coronal and middle thirds of root canals were prepared with \#2, \#3, and \#4 Gates Glidden burs (Dentsply Maillefer). Intermittent irrigation was performed with $5.25 \%$ sodium hypochlorite solution. Canal patency was checked with \#06, \#08, and \#10 K-files (Dentsply Maillefer) that were passed through the apex. Excess fluid was removed from the pulp chamber, but no attempt was made to dry the canal. Teeth were measured with a \#10 K-file introduced into the canal until the tip was just visualized by means of a microscope (Carl Zeiss GmbH, Oberkochen, Germany) at $5 \times$ magnification at the level of the most coronal border of the major foramen (MF) $(28,29)$. When the file tip was observed at the MF, the silicone ring was stabilized at the coronal edge of the tooth, the file was removed, and the distance between the stop and the file tip was measured with a digital caliper (MitutoYo 571-202-20; Mitutoyo Italiana SRL, Linate, Italy). We recorded this value as the initial working length (iWL). Specimens were then placed in a tooth holder, which is part of an endodontic training kit (Pro-Train; Simit Dental, Mantova, Italy), and tightly held to prevent displacement during procedures. Canals were then shaped with ProTaper Universal instruments (Dentsply Maillefer) as per the manufacturer's instructions until F2 reached the iWL. Canals were irrigated after each file was used with $10 \mathrm{~mL} 5.25 \%$ sodium hypochlorite delivered with a 27-G needle (Nipro, Osaka, Japan).

\section{Direct Length Measurements}

Once the canal preparation was complete, teeth were withdrawn from the Pro-Train and directly measured as described for the iWL. These measurements were noted as the direct length (DL) and became our reference point for the comparison of electronic measurements.

\section{Electronic Length 1 Measurements}

To record the electronic measurements, we used the Pro-Train as described by Cianconi et al (28), and the specimens were placed once again in the tooth holder. Electronic measurements were obtained with the Root ZX unit EAL (J Morita Corp, Kyoto, Japan). Canals were irrigated with $5 \mathrm{~mL}$ 5.25\% sodium hypochlorite, and excess solution was removed with absorbent paper points. A \#25 K-file was used to obtain electronically determined WL readings. The K-file was advanced until the EAL display was flashing the word "APEX" (28-34). A rubber stop was then carefully adjusted to the reference level, and the file was withdrawn for measurement of the distance between the rubber stop and the file tip by means of a digital caliper (MitutoYo 571-20220). These measurements were referred to as electronic length 1 (EL1).

\section{Root Canal Filling}

Canals were then irrigated with $2 \mathrm{~mL} 5.25 \%$ of sodium hypochlorite, dried with absorbent paper points, and divided into 2 groups: ProTaper Obturator $(n=25)$ and GuttaCore $(n=25)$. In both groups, apical canal diameters were assessed, respectively, with a ProTaper Obturator size verifier and a GuttaCore size verifier. Canals were coated with a thin layer of AH Plus root canal sealer (Dentsply DeTrey, Konstanz, Germany) by means of paper points. The obturators recommended for a size \#25 .08 taper preparation are a ProTaper F2 Obturator and a size 25 GuttaCore Obturator. Obturators were softened in the ThermaPrep Oven (Dentsply Maillefer) according to the manufacturer's instructions and inserted up to $0.5 \mathrm{~mm}$ to DL. The obturators were cut at the orifice with a Thermacut bur (Dentsply Maillefer), and the canal fillings were then compacted vertically at the orifice. After a small cotton pellet was placed at the canal orifice and the access cavity was sealed with Cavit 3M (Henry Schein, New York, NY), the teeth were stored in $100 \%$ humidity for 7 days to ensure setting of the sealer.

\section{Electronic Length 2 Measurements}

After 7 days, the temporary restoration was removed. The coronal and middle thirds of root canal obturation were removed with \#2, \#3, and \#4 Gates-Glidden burs. The samples were then again placed in the Pro-Train. The apical third of the root filling was softened with a drop of a gutta-percha solvent (Endosolv E; Septodont, Saint-Maur-des-Fossès Cedeux, France). For the electronically determined length, a \#25 Kfile was attached to the clip of Root ZX. The file was gently inserted into the softened apical gutta-percha until the "APEX" signal was emitted. The rubber stop was then carefully adjusted to the reference level, and the file was withdrawn for measurement of the distance between the rubber stop and the file tip with the digital caliper (MitutoYo 571-202-20). These measurements were recorded as electronic length 2 (EL2).

\section{Evaluation Griteria and Statistical Analysis}

DL values were recorded by 1 experienced endodontist. EL1 and EL2 values for each sample were recorded by 2 experienced and standardized endodontists, and the highest value was taken. The difference between the measurements taken by the 2 operators was always less than $1 \%$. All data were initially entered into an EXCEL database (Microsoft, Redmond, WA), and analysis was performed with the Statistical Package for the Social Sciences for Windows (version 15.0; SPSS, Chicago, IL). Descriptive statistics consisted of the mean \pm standard deviation for parameters with Gaussian distributions (after confirmation with histograms and the Kolmogorov-Smirnov test). Electronic measurements before and after root filling (EL1 and EL2) were compared with the original direct measurements (DL). When DL was greater than EL1 and/or EL2, the difference was negative, indicating that, in the electronic measurement, the file tip was inside the canal and did not reach the foramen. When DL was smaller than EL1 and/or EL2, the difference was positive, indicating that the file tip was outside the canal, beyond the 
TABLE 1. Differences between the Actual Root Canal Length Obtained by the Direct Method and the Working Length Obtained by the Electronic Method before and after Root Canal Filling

\begin{tabular}{|c|c|c|c|c|c|c|c|c|c|c|c|}
\hline Tratt & Measure & WLI & DL & EL1 & EL2 & $\begin{array}{l}\text { Diff EL1 } \\
\text { (EL1-DL) }\end{array}$ & $\begin{array}{l}\text { Diff EL2 } \\
\text { (EL2-DL) }\end{array}$ & $\begin{array}{c}\text { Ratio EL1 } \\
\text { EL1/DL }\end{array}$ & $\begin{array}{c}\text { Mean } \\
\text { EL1 }\end{array}$ & $\begin{array}{c}\text { Ratio EL2 } \\
\text { EL2/DL }\end{array}$ & $\begin{array}{c}\text { Mean } \\
\text { EL2 }\end{array}$ \\
\hline \multirow[t]{5}{*}{1} & $n$ & 25 & 25 & 25 & 25 & 25 & 25 & 25 & 25 & 25 & 25 \\
\hline & Mean & 16.64 & 16.60 & 16.82 & 17.00 & 0.22 & 0.40 & 1.01 & 16.71 & 1.02 & 16.80 \\
\hline & SD & 0.60 & 0.56 & 0.518 & 0.56 & 0.29 & 0.41 & 0.02 & 0.52 & 0.03 & 0.52 \\
\hline & Minimum & 15.5 & 15.5 & 16.0 & 16.0 & 0.0 & 0 & 1.0 & 16.0 & 1.0 & 16.0 \\
\hline & Maximum & 18.0 & 18.0 & 18.0 & 18.0 & 1.0 & 1 & 1.1 & 18.0 & 1.1 & 18.0 \\
\hline \multirow[t]{5}{*}{2} & $n$ & 25 & 25 & 25 & 25 & 25 & 25 & 25 & 25 & 25 & 25 \\
\hline & Mean & 16.12 & 16.06 & 16.16 & 16.46 & 0.10 & 0.40 & 1.01 & 16.11 & 1.03 & 16.26 \\
\hline & SD & 0.67 & 0.63 & 0.67 & 0.59 & 0.20 & 0.30 & 0.01 & 0.65 & 0.02 & 0.60 \\
\hline & Minimum & 14.5 & 14.5 & 14.5 & 15.0 & 0.0 & 0 & 1.0 & 14.5 & 1.0 & 14.8 \\
\hline & Maximum & 17.5 & 17.5 & 17.5 & 17.5 & 0.5 & 1 & 1.0 & 17.5 & 1.1 & 17.5 \\
\hline \multirow[t]{5}{*}{ Total } & $N$ & 50 & 50 & 50 & 50 & 50 & 50 & 50 & 50 & 50 & 50 \\
\hline & Mean & 16.38 & 16.33 & 16.49 & 16.73 & 0.16 & 0.40 & 1.01 & 16.41 & 1.03 & 16.53 \\
\hline & SD & 0.68 & 0.65 & 0.68 & 0.63 & 0.26 & 0.35 & 0.02 & 0.65 & 0.02 & 0.62 \\
\hline & Minimum & 14.5 & 14.5 & 14.5 & 15.0 & 0.0 & 0 & 1.0 & 14.5 & 1.0 & 14.8 \\
\hline & Maximum & 18.0 & 18.0 & 18.0 & 18.0 & 1.0 & 1 & 1.1 & 18.0 & 1.1 & 18.0 \\
\hline
\end{tabular}

Diff, difference; DL, direct length; EL, electronic length; SD, standard deviation; Tratt, treatment group; WL, working length.

foramen. The means of differences between EL1/DL and EL2/DL were calculated and compared using the Bland and Altman method. A $P$ value of $<.05$ was considered statistically significant. The difference between EL1 and EL2 and the different influences of the 2 obturating materials on the accuracy of the EAL were calculated with repeated measures analysis of variance.

\section{Results}

The differences between the actual root canal length obtained by the direct method and the WLs obtained by the electronic method before and after root canal filling (DL/EL1 and DL/EL2) are shown in Table 1, which clearly shows that EL1 and EL2 values are close to DL with EL1 closer. Moreover, the mean of the differences (diffEL1 = EL1-DL and diffEL2 = EL2-DL) gives positive values, indicating that both EL1 and EL2 tend to overestimate the actual root canal length, with EL2 higher from DL.

Figure 1 shows that both EL1 and EL2 give a statistically significant overestimation of the actual root canal length (DL). Figure 2 shows that the ratioEL1 (EL1/DL) and the ratioEL2 (EL2/DL) are significantly different from each other $(P<.0001)$, indicating that EL1 and EL2 are also significantly different from each other. The 2 different treatments do not influence the accuracy of the EAL differently $(P=.486)$.

\section{Discussion}

A main role for primary endodontic treatment and orthograde retreatment is the chemomechanical preparation and the 3-dimensional obturation of the root canal system (35). The accurate determination of and respect for the correct WL are a crucial aspect of orthograde endodontic retreatments (35). EALs are useful instruments for correctly locating the root canal length in primary root canal treatment (17) as well as in orthograde retreatment (20). Although the most recent EALs can provide accurate measurements, even in the presence of irrigating solutions and/or pus (36), it has been shown that endodontic content can influence their accuracy $(23,37)$. Al-Bulushi et al (24) reported that root canal obturation could influence root canal impedance. Therefore, during retreatment, the presence of obturation materials, as well as solvents, could influence EAL readings. This assumes greater importance if we consider that it has been widely demonstrated that it is almost impossible to obtain the complete removal of guttapercha and/or sealer from the endodontic space during orthograde retreatment (38).
In the present study, we evaluated the influence of 2 different obturation systems (ProTaper Obturator and GuttaCore) on the accuracy of Root ZX. Models in which extracted teeth are immersed in media with electrical resistance similar to that of the periodontal tissue can give precise information on EAL accuracy (27). In the present study, an ecoelectro-conductive gel was used as a medium because of its suitable electro-conductive properties, which Cianconi et al (28) showed to be of better quality compared with those of other materials. In accordance with reports from other studies $(2,20)$, DL of canals was obtained after canals were shaped because tooth length might be modified. In accordance with many other studies (28-34) but in contrast with the manufacturer's suggestion, the "APEX" mark of the EAL was used to measure electronic lengths. This decision was taken to avoid bias calculating the difference between DL and electronic measurements (EL1, EL2). In fact, in our experimental procedure, DL was measured at the MF because it has been shown that only the "APEX" mark represents the MF consistently $(22,34,39)$, whereas the " 0.5 " mark simply represents an area slightly short of the MF, which might result in inconsistent measurements (34). Root ZX is not able to detect $0.5 \mathrm{~mm}$ from the foramen and should only be used to detect the MF (22), which is better to test for EAL accuracy (40). We compared DL values with both EL1 and EL2 values. From the mean of the differences, it was clear that EL1 values overestimated DL values. The mean overestimation for group 1 was $0.22 \mathrm{~mm}$, whereas that in group 2 was $0.10 \mathrm{~mm}$. For all samples, the mean overestimation was $0.16 \mathrm{~mm}$. The maximum overestimation found was $1 \mathrm{~mm}$. In no sample was the EL1 value found to be lower than the respective DL value. Although EALs are considered accurate in determining root canal length, many studies demonstrated that, when the sign "APEX" appears, the mean distance between the file tip and the apical constriction always has positive values (25), indicating that, normally, EALs give a slight overestimation of actual root canal length. Thus, our findings are in accordance with those of other authors $(2,25,28,29)$.

In contrast to our findings, Alves et al (20) and Ebrahim et al (27) showed that EL1 values tended to underestimate root canal length. Alves et al hypothesized that this result is caused by the presence of debris and dentin chips created during shaping because, in accordance with our work, measurements were obtained after preparation. In the study by Ebrahim, the results can be explained by the fact that the author did not recheck the WL after shaping. Thus, a hypothetical shortening of the root canal length could have caused the underestimation. In the present study, during the retreatment procedures, the apex was 


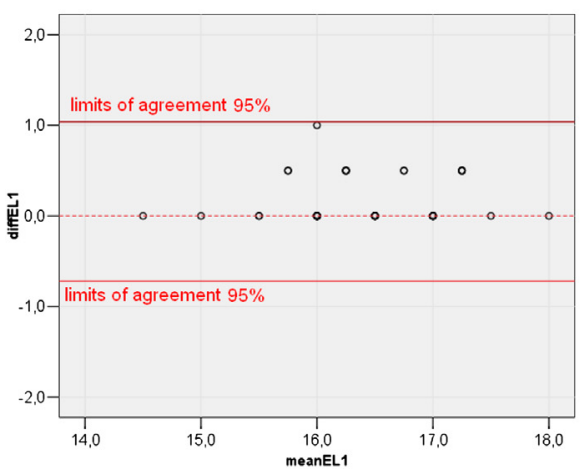

Figure 1. Ninety-five percent limits of agreement of Bland and Altman.

always reached in all samples. This result was achieved with the use of a solvent. In fact, solvents may facilitate the removal of root canal content (23), and, when a retreatment is performed without solvent, it can be more difficult to negotiate the apex again (2). From the mean of the differences (EL2-DL), it is clear that the EL2 value also tended to overestimate the DL value. The mean overestimation of the actual root canal length was $0.40 \mathrm{~mm}$ in both groups 1 and 2 . Thus, $0.40 \mathrm{~mm}$ was also the mean overestimation in all samples. The maximum overestimation found was $1 \mathrm{~mm}$, whereas in no samples was EL2 lower than DL. The mean of the EL1-DL differences was lower than the mean of the EL2DL differences, indicating that EL1 is closer to DL than EL2. Thus, the EAL is less accurate during retreatment. It should be noted that the differences between measurements showed a trend toward overestimation for both EL1 and EL2. No EL1 and EL2 values gave an underestimation of the actual root canal length. In accordance with our findings, Alves et al (20) and Aggarwal et al (2) obtained an overestimation of the actual root canal length by the EL2 values even though, in contrast to our findings, Alves et al obtained EL2 values closer to DL compared with EL1 values. This can be explained by the fact that in his study EL1 values gave an underestimation of DL. Thus, during retreatment,

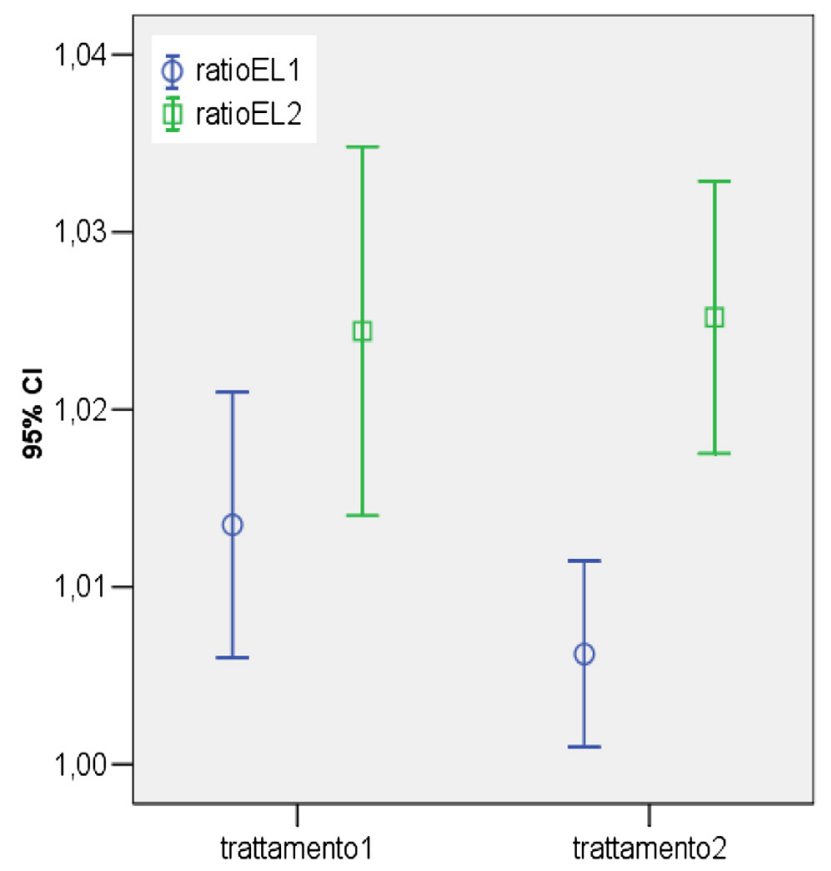

Figure 2. Analysis of variance 1-way vs treatments. CI, confidence interval.

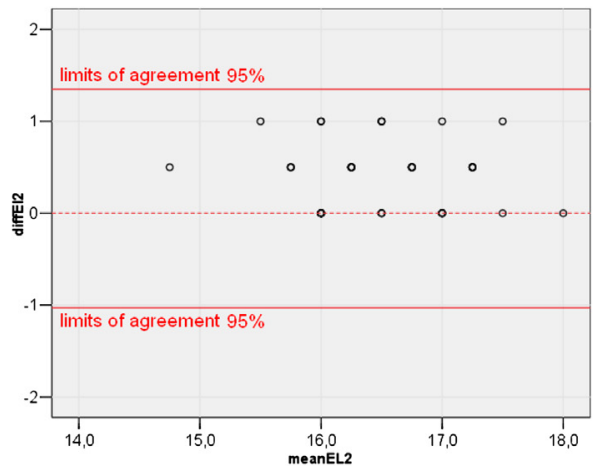

because the file had to pass through the gutta-percha to give an electric signal, EL2 values were higher than EL1 values and, therefore, were closer to DL values (20).

In contrast to our results, Ebrahim et al (27) found that EL2 values tended to underestimate DL values. Once again, this can be explained by the fact that the author did not consider the working length after primary root canal instrumentation. In our study, we also found a significant difference between EL1 and EL2 $(P<.0001)$, with EL2 values higher than those for EL1. These findings are in accordance with those shown by other authors (20). In contrast to our findings, Er et al (23) obtained lower EL2 than EL1 values. They hypothesized that the electrical conductivity of solvents could explain the poor accuracy of EALs. According to our experimental protocol, this result can also be explained by the fact that the authors did not verify the WL after primary canal shaping; moreover, they calculated the retreatment $\mathrm{WL}$ after the complete removal of endodontic content and the additional enlargement of root canals beyond the initial canal preparation. Thus, a presumable shortening of the canal could explain their results. In our study, the 2 different root canal obturating materials were tested for their different influence on the accuracy of the EAL, but no statistically significant differences were found.

From our findings, it can be hypothesized that overestimation indicated by EL2 values can be explained by the apical extrusion of the endodontic obturating material during the retreatment procedures that, because of poor conductivity, can interfere with the electronic readings. It should be noted that the presence of solvent could also affect the accuracy of the tested EAL as shown in a recent study (23). In fact, the electrical conductivity of solvents could be responsible for the poor accuracy of EALs (23). Thus, it can be hypothesized that, in vivo, there can be an even higher overestimation because favorable circumstances for precise measurements are never available. Moreover, it should be noted that our results were obtained with formed and sound apices. In vivo, clinicians often perform orthograde retreatment in teeth with resorbed apices caused by chronic apical inflammation. In these cases, the risk for overestimation of the actual root canal length is even greater if clinicians do not calculate ex novo the WL after the shaping.

\section{Conclusions}

We found the following:

1. The 2 electronic measurements (EL1 and EL2) give an overestimation of the actual WL (ie, DL).

2. The 2 different carrier-based obturating materials have a statistically significant influence on the accuracy of the EAL, but there was no statistically significant difference between them. 
Based on the results of the present study and using the EAL tested, to prevent overestimation of root canal length in orthograde retreatments, clinicians should subtract at least $1 \mathrm{~mm}$ from the WL measurement on the APEX mark before shaping and sealing the root canal system. In any case, for successful orthograde retreatment, it is essential to recalculate the root canal length after the complete removal of endodontic material.

\section{Acknowledgments}

The authors thank Dr Roberto Sorge for assisting with the statistical analysis.

The authors deny any conflicts of interest related to this study.

\section{References}

1. Green D. Stereomicroscopic study of 700 root apices of maxillary and mandibular posterior teeth. Oral Surg Oral Med Oral Pathol 1960;13:728-33.

2. Aggarwal V, Singla M, Kabi D. An in vitro evaluation of performance of two electronic root canal length measurement devices during retreatment of different obturating materials. J Endod 2010;36:1526-30.

3. Barbizam JVB, Fariniuk LF, Marchesan MA, et al. Effectiveness of manual and rotary instrumentation techniques for clearing flattened root canals. J Endod 2002;28: $365-6$

4. De Cleen M, Schuurs A, Wesselin KP, Wu M. Periapical status and prevalence of endodontic treatment in an adult Dutch population. Int Endod J 1993;26:112-9.

5. Taintor J, Ingle J, Fahid A. Retreatment versus further treatment. Clin Prev Dent 1983:5:8-14.

6. Lovdahl P, Gutmann J. Problems in nonsurgical root canal retreatment. In: Gutmann J. Dumsha T, Lovdahl P, eds. Problem Solving in Endodontics. 3rd ed. St. Louis, MO: Mosby; 1993:157-202.

7. Gorni FG, Gagliani MM. The outcome of endodontic retreatment: a 2-year follow-up. J Endod 2004:30:1-4.

8. Whitwhorth J. Methods of filling root canals: principles and practices. Endod Topics 2005;12:2-24

9. Duncan HF, Chong BS. Removal of root filling materials. Endod Topics 2011;19: $33-57$

10. Johnson WB. A new gutta-percha technique. J Endod 1978;4:184-8.

11. Sutow EJ, Foong W-CH, Zakarisen KL, et al. Corrosion and cytotoxicity evaluation of Thermafil endodontic obturator carriers. J Endod 1999;25:562-6.

12. Wilkox LR, Juhlin JJ. Endodontic retreatment of Thermafil versus laterally condensed gutta-percha. J Endod 1994;20:115-7.

13. Baratto Filho F, Ferreira EL, Fariniuk LF. Efficiency of the 0.04 taper ProFile during the re-treatment of gutta-percha-filled root canals. Int Endod J 2002;35:651-4.

14. GuttaCore Brochure (2011) Dentsply Tulsa Dental Specialties. Available at: http:// www.tulsadental.com/lit2/pdfs/BRGC12-10_GuttaCore_broch_Lo.pdf. Accessed July $18,2014$.

15. Bergenholtz D, Lekholm U, Milthon R, Engstrim B. Influence of apical overinstrumentation and overfilling on re-treated root canals. J Endod 1979;5:310-4.

16. Goldberg F. Evaluacion clinica del Root ZX en la determinacion de la conductometria. Rev Asoc Odontol Argent 1995;83:180-2.

17. Shabahang S, Goon WWY, Gluskin AH. An in vivo evaluation of Root ZX electronic apex locator. J Endod 1996;22:616-8.
18. Dunlap CA, Remeikis NA, BeGole EA, Rauschenberg CR. An in vivo evaluation of an electronic apex locator that uses the ratio method in vital and necrotic canals. J Endod 1998;24:48-50.

19. El Ayouti A, Weiger R, Lost C. The ability of Root ZX apex locator to reduce the frequency of overestimated radiographic working length. J Endod 2002;28:116-9.

20. Alves AM, Felippe MC, Felippe WT, Rocha MJ. Ex vivo evaluation of the capacity of the Tri Auto ZX to locate the apical foramen during root canal retreatment. Int Endod J 2005;38:718-24

21. Ibarrola JL, Chapman BL, Howard JH, et al. Effect of preflaring on Root ZX apex locators. J Endod 1999;25:625-6.

22. Ounsi HF, Naaman A. In vitro evaluation of the reliability of the Root-ZX electronic apex locator. Int Endod J 1999;32:120-3.

23. Er 0, Uzun 0, Ustun Y, et al. Effect of solvents on the accuracy on the Mini Root ZX apex locator. Int Endod J 2013;46:1088-95.

24. Al-Bulushi A, Levinkind M, Flanagan M, et al. Effect of canal preparation and residual root filling material on root impedance. Int Endod J 2008;41:892-904.

25. Nekoofar MH, Ghandi MM, Hayes SJ, Dummer PM. The fundamental operating principles of electronic root canal length measurement devices. Int Endod J 2006;39: 595-609.

26. Goldberg F, Marroquin BB, Frajlich S, Dreyer C. In vitro evaluation of the ability of three apex locators to determine the working length during retreatment. J Endod 2005:31:676-8.

27. Ebrahim AK, Wadachi R, Suda H. In vitro evaluation of the accuracy of five different electronic apex locators for determining the working length of endodontically retreated teeth. Aust Endod J 2007;33:7-12.

28. Cianconi L, Angotti V, Felici R, et al. Accuracy of three electronic apex locators compared with digital radiography; an ex vivo study. J Endod 2010;36:2003-7.

29. Mancini M, Felici R, Conte G, et al. Accuracy of three electronic apex locators in anterior and posterior teeth: an in vivo study. J Endod 2011;37:684-7.

30. Guise GM, Goodell GG, Imamura GM. In vitro comparison of three electronic apex locators. J Endod 2010;36:279-81.

31. Piasecki L, Carneiro E, Fariniuk LF, et al. Accuracy of Root ZX II in locating foramen in teeth with apical periodontitis: an in vivo study. J Endod 2011:37:1213-6.

32. Herrera M, Abalos C, Planas AJ, Llamas R. Influence of apical constriction diameter on Root ZX apex locator precision. J Endod 2007;33:995-8.

33. Janner SFM, Jeger FB, Lussi A, Bornstein MM. Precision of endodontic working length measurements: a pilot investigation comparing cone-beam computed tomography scanning with standard measurement techniques. J Endod 2011;37:1046-51.

34. Jung I-Y, Yoon B-H, Lee S-J, Lee SJ. Comparison of the reliability of " 0.5 " and "APEX" mark measurements in two frequency-based electronic apex locators. J Endod 2011:37:49-52.

35. Ricucci D, Langeland K. Apical limit of root canal instrumentation and obturation, part 2: a histological study. Int Endod J 1998;31:394-409.

36. Haffner C, Folwaczny M, Galler K, Hickel R. Accuracy of electronic apex locators in comparison to actual length: an in vivo study. J Dent 2005:33:619-25.

37. Goldberg F, De Silvio AC, Manfre S, et al. In vitro measurement accuracy of an electronic apex locator in teeth with simulated apical root resorption. J Endod 2002;28: $461-3$.

38. Zmener 0, Pameijer CH, Banegas G. Retreatment efficacy of hand versus automated instrumentation in oval-shaped root canals: an ex vivo study. Int Endod J 2006;39: $521-6$.

39. Mayeda DL, Simon JH, Aimar DF, et al. In vivo measurement accuracy in vital and necrotic canals with the Endex apex locator. J Endod 1993;19:545-8

40. Lee SJ, Nam KC, Kim YJ, et al. Clinical accuracy of a new apex locator with an automatic compensation circuit. J Endod 2002;28:706-9. 